\title{
Ceruloplasmin dysfunction and therapeutic potential for Parkinson's disease
}

Running head: Ceruloplasmin in Parkinson's disease

Scott Ayton $\mathrm{PhD}^{1}$, Peng Lei $\mathrm{PhD}^{1}$, James A. Duce, $\mathrm{PhD}^{1}$, Bruce X. W. Wong, $\mathrm{PhD}^{1}$, Amelia

Sedjahtera BBMS(Hons) ${ }^{1}$, Paul A. Adlard $\mathrm{PhD}^{2,3}$, Ashley I. Bush MBBS $\mathrm{PhD}^{1,3,{ }^{*}}$ and David I.

Finkelstein, $\mathrm{PhD}^{1,2}$

${ }^{1}$ Oxidation Biology Laboratory and ${ }^{2}$ Synaptic Neuropathology, Florey Institute for Neuroscience and Mental Health, and ${ }^{3}$ Department of Pathology, The University of Melbourne, Victoria, Australia.

*Correspondence to: ashleyib@unimelb.edu.au

Words:

-Abstract: 97 words

-Body: 2112 words

3 Main Figures, 1 Supplementary Table and 3 Supplementary Figures

\section{Abstract}

Ceruloplasmin is an iron-export ferroxidase that is abundant in plasma and also expressed in glia.

We found a $\sim 80 \%$ loss of ceruloplasmin ferroxidase activity in the substantia nigra of idiopathic

Parkinson's disease cases, which could contribute to the pro-oxidant iron accumulation that

characterizes the pathology. Consistent with a role for ceruloplasmin in PD etiopathogensis, ceruloplasmin knock-out mice developed parkinsonism that was rescued by iron chelation.

Additionally, peripheral infusion of ceruloplasmin attenuated neurodegeneration and nigral iron elevation in the MPTP mouse model for PD. These findings show, in principle, that intravenous ceruloplasmin may have therapeutic potential in PD.

This article has been accepted for publication and undergone full peer review but has not been through the copyediting, typesetting, pagination and proofreading process which may lead to differences between this version and the Version of Record. Please cite this article as an 'Accepted Article', doi: 10.1002/ana.23817 


\section{Introduction}

Iron accumulation in the substantia nigra (SN) is a feature of Parkinson's disease (PD) $)^{1,2}$, where the iron burden could cause oxidative stress ${ }^{3}$ and contribute to Lewy body pathology ${ }^{4,5}$. Iron chelation, by drugs such as deferiprone ${ }^{6}$, is a prominent therapeutic strategy for PD. Nigral iron accumulation could be contributed by a disturbance of iron export. The multi-copper ferroxidase, ceruloplasmin, facilitates cellular iron export by oxidizing $\mathrm{Fe}^{2+}$ presented by ferroportin at the cell surface for incorporation into the extracellular iron transporter, transferrin ${ }^{7}$.

Within the brain, ceruloplasmin is expressed in glia $^{8}$, and we characterized the $\beta$-amyloid precursor protein (APP) as the ferroxidase of neurons ${ }^{9}$. We showed recently that tau protein, required for trafficking of APP to the neuronal surface, is decreased in the nigra in $\mathrm{PD}^{2}$, and could contribute to pathogenic iron elevation in neurons ${ }^{1}$. The cause of iron elevation in nigral glia in $\mathrm{PD}^{1}$ is unknown.

Despite not being expressed in neurons ${ }^{8}$, functional depletion of ceruloplasmin causes neurodegeneration. Aceruloplasminemia, an autosomal recessive disorder where mutations abolish functional ceruloplasmin, commonly present with variegated neurological phenotypes including parkinsonism, cognitive loss, cerebellar and retinal degeneration ${ }^{10}$. Carrying one mutant ceruloplasmin allele increases the risk for $\mathrm{PD}^{11}$. Analogously, ceruloplasmin knockout $\left(\mathrm{CP}^{-/-}\right)$mice develop nigral and cerebellar iron accumulation, with neurodegeneration and motor deficits $^{12}$. Decreased ceruloplasmin ferroxidase activity in $\mathrm{CSF}^{13-15}$ and serum ${ }^{16-21}$ from idiopathic PD patients has been reported, and decreased serum ceruloplasmin levels is associated with earlier age of PD-onset ${ }^{17,19,20}$. The status of ceruloplasmin in PD brain tissue is hitherto unknown. We hypothesized that in PD, decreased serum and CSF derived ceruloplasmin activity may reflect changes in brain ceruloplasmin that could engender vulnerability to iron, and, if so, 
communication between brain and peripheral pools of ceruloplasmin might be a portal for treatment by peripheral infusion of ceruloplasmin.

\section{Methods}

\section{Human brain}

Post-mortem brain tissue was obtained from the Victorian Brain Bank Network. Tissue from 10 control individuals (5 male, $76.6+/-2.1$ years) and 10 age-matched patients with clinically and pathologically defined PD (6 male, 75 +/- 2.0 years; Supplementary Table 1) was homogenized by pulse sonication in PBS with protease inhibitors (Pierce) and phosphatase inhibitor cocktails $1 \& 2$ (Sigma).

\section{$\underline{\text { Mice }}$}

At the conclusion of each experiment below, mice were transcardially perfused with ice-cold PBS under deep anaesthesia (sodium pentobarbitone, $100 \mathrm{mg} / \mathrm{kg}$ ).

Deferiprone treatment: Deferiprone (Sigma Medical) was administered in drinking water of 3months-old $\mathrm{CP}^{-/-}$mice $(1 \mathrm{mg} / \mathrm{ml})$ for three months; these were compared to age-matched untreated $\mathrm{CP}^{-/-}$and WTs. Mice on average drank $3 \mathrm{ml}$ of deferiprone-water per day; the dose of deferiprone ingested was approximately $100 \mathrm{mg} / \mathrm{kg} /$ day. Before death, mice underwent Rotarod, Open Field, and Y-maze tasks (Supplementary Methods). Left SN was dissected for biochemical analysis, and right $\mathrm{SN}$ was sectioned for stereology.

MPTP: C57/B16 administered MPTP (4x10mg/kg, IP, 2 hours apart), were euthanized after 21 days. Micro-dissected SN was homogenized as above.

Ceruloplasmin treatment to $C P^{-/-}$mice: Human ceruloplasmin (Vital products) was administered 
to $\mathrm{CP}^{-/-}$mice $(5 \mathrm{mg} / \mathrm{kg}$ in saline, I.P.) and separate groups of mice were euthanized at time-points indicated. Whole brains were homogenized as above.

MPTP rescue with ceruloplasmin: Two groups of 3-month-old C57/B16 mice were administered MPTP (as above) and a third group of untreated mice was used as controls. One group received ceruloplasmin protein $(5 \mathrm{mg} / \mathrm{kg}$ in saline, IP) 24 hours and 48 hours after MPTP, then every third day thereafter. The second MPTP-group was administered saline. Mice were euthanized 21 days after MPTP. Left SN was homogenized as above, and right SN was sectioned for stereology.

Ceruloplasmin ferroxidase activity (whole tissue homogenate)

Samples were assayed using established protocols ${ }^{9}$. The ceruloplasmin ferroxidase assay, which we adapt to PD tissue, was first described in 1966 (Osaki et al, JBC, 1996). The assay requires iron, apo-transferrin and holo-ceruloplasmin (copper loaded); oxygen is also consumed in the reaction. The premise of the assay is that when the added ferrous iron is oxidized by ceruloplasmin, the ferric iron species is then able to load into transferrin, which causes a color change with peak absorbance of 460nm. Ceruloplasmin is selectively inhibited by sodium azide (Osaki et al, JBC, 1996). The experiment is performed in duplicate: $+/-20 \mathrm{mM}$ sodium azide (Sigma). Oxidation by ceruloplasmin in the sample is calculated by subtracting the azide inhibited reading from the original sample reading. A sample blank is also subtracted from both readings to control for background oxidation of ferrous iron. To prepare the brain samples, Triton X-100 (0.1\%) was added to PBS homogenized samples, and the soluble fraction was taken after ultracentrifugation $\left(100,000 \mathrm{~g}\right.$ for 30 minutes at $\left.4^{\circ} \mathrm{C}\right)$. Samples of soluble extract (30 $\mu \mathrm{g}$ protein) were incubated $\left(5 \mathrm{~min}, 37^{\circ} \mathrm{C}\right.$ with agitation) with $75 \mathrm{mM}$ sodium acetate $\mathrm{pH} 6.0,55$ $\mu \mathrm{M}$ apo-transferrin (Sigma), $110 \mu \mathrm{M}$ ferrous ammonium sulfate (Sigma) $\pm 20 \mathrm{mM}$ sodium azide (Sigma), before absorbance was measured in a $1 \mathrm{ml}$ cuvette $(460 \mathrm{~nm})$. 
Ceruloplasmin ferroxidase activity (ceruloplasmin immunodepleted homogenate)

Brain samples were homogenized by pulse sonication in TBS with $0.1 \%$ Triton $\mathrm{X}-100(\mathrm{pH}$

7.4). $1 \mathrm{mg}$ of protein from each brain homogenate was pre-cleared in the presence of Protein $\mathrm{G}$

Sepharose beads (Invitrogen). The cleared supernatant was then added to fresh beads; 1 aliquot

also contained $10 \mu \mathrm{l}$ anti-ceruloplasmin antibody (Vital Diagnostics) while the other aliquot was used as an antibody control. Samples were incubated overnight $\left(4^{\circ} \mathrm{C}\right)$, then centrifuged at 5000

RPM for 2 minutes. The supernatant was then probed for ceruloplasmin by western blot to ensure immunodepletion. Samples were assayed by a modified transferrin-ferroxidase assay. 50 $\mu \mathrm{g}$ of supernatant was added to $50 \mathrm{mM}$ HEPES, $150 \mathrm{mM} \mathrm{NaCl}, 55 \mu \mathrm{M}$ apo-transferrin, and 110 $\mu \mathrm{M}$ ferrous ammonium sulfate $\left(\mathrm{pH}\right.$ 7.4). Samples were incubated at $26^{\circ} \mathrm{C}$ for 10 minutes and absorbance was recorded at $460 \mathrm{~nm}$ on a 96-well plate. A condition without sample homogenate was used to correct background ferrous oxidation. The background oxidation signal was subtracted from the signal recorded in the homogenate samples. Ferroxidase activity of ceruloplasmin was determined by subtracting the ferroxidase signal of the sample aliquot that had ceruloplasmin immunodepleted from the sample aliquot that was not exposed to ceruloplasmin immunodepletion (sham immunodepleted).

$\underline{\text { Additional Methods }}$

Detailed methodology is described in the Supplementary Methods.

\section{Results}

Decreased nigral ceruloplasmin activity accompanies iron accumulation in Parkinson's Disease

Relative to levels from age-matched control samples $(n=10)$, PD-affected SN tissue $(n=10)$ exhibited iron elevation $(+42 \%, P=0.021)$ and decreased copper $(-51 \%, P=0.003$; Fig. 1A). 
Despite no change in ceruloplasmin protein levels in PD SN, ceruloplasmin activity (as determined by change in signal upon azide inhibition) was markedly decreased $(-80 \%, P<0.001$;

Fig. 1A). Ceruloplasmin levels and activity were not associated with post mortem interval (PMI) of the subjects (Supplementary Figure 1). To further establish that ceruloplasmin activity is lost in PD SN, we immunodepleted ceruloplasmin from SN brain homogenate in a subset of control $(n=5)$ and PD ( $n=7)$ subjects. Ceruloplasmin ferroxidase activity was determined by the subtraction of the ferroxidase signal of the immunodepleted sample from the sham immunodepleted sample. By this method, decreased ceruloplasmin ferroxidase activity was again observed in PD SN compared to control $(-75 \%, P=0.03$; Fig. 1B). No changes in iron, copper, ceruloplasmin protein or activity were observed comparing PD-affected to normal age-matched frontal cortex tissue samples (Fig. 1C), revealing tissue specific association between the loss of ceruloplasmin activity and iron elevation.

\section{$\underline{\text { Ceruloplasmin ablation results in iron-mediated Parkinsonism }}$}

If reduced ceruloplasmin activity in PD-affected SN contributes to neurodegeneration via iron accumulation, then iron chelation should rescue the Parkinson pathology that develops in $\mathrm{CP}^{-/-}$mice ${ }^{12}$. Six-months-old WT mice $(\mathrm{n}=10)$ were compared with age-matched untreated $\mathrm{CP}^{-/-}$ mice $(n=8)$, and age-matched $\mathrm{CP}^{-/-}$mice $(n=9)$ that were treated for 3 months with the chelator, deferiprone. At the end of this period, Rotarod challenge revealed that $\mathrm{CP}^{-/-}$mice withstood significantly less time upon the rod $(-40 \% ; P=0.043)$, but this disability was rescued by deferiprone ( $P=0.02$; Fig. 2A). Similarly, survival curves of increasing Rotarod speed showed that the untreated $\mathrm{CP}^{-/-}$mice fell at a lesser speed then did WT mice $(P=0.025)$, while deferiprone-treated $\mathrm{CP}^{-/-}$mice were indistinguishable from control (Fig. 2B).

Consistent with pathogenesis being mediated by elevated iron, nigral iron levels were 
significantly elevated in $\mathrm{CP}^{-/-}$mice $(+46 \% ; P=0.008)$, but this was normalized by deferiprone treatment $(P=0.007$; Fig. 2C). Accordingly, the loss of SN neurons observed in the non-treated $\mathrm{CP}^{-/-}$mice $(P<0.001)$, was partially rescued by deferiprone $(P=0.002$; Fig. 2D,E).

Rotarod disability is associated with SN neuron $\operatorname{loss}^{22}$; however extra-nigral degeneration could also cause such disability. We investigated if other faculties were also affected in $\mathrm{CP}^{-/-}$ mice. In the Y-maze challenge, there was no difference in the time spent in the novel arm between WT, $\mathrm{CP}^{-/-}$, and $\mathrm{CP}^{-/-}$-treated mice (Supplementary Fig. 2A), indicating that $\mathrm{CP}^{-/-}$are not cognitively impaired at this age. These mice were also not affected in the Open Field assay, demonstrating comparable general health and arousal (Supplementary Fig. 2B-E). The behavior data were consistent with anatomical characterization where we observed no change in the size of the lateral ventricle, or cortical thickness in $\mathrm{WT}, \mathrm{CP}^{-/-}$, and $\mathrm{CP}^{-/-}$-treated mice at 6-months of age (Supplementary Fig. 3).

MPTP decreases nigral ceruloplasmin levels

We investigated changes in nigral ceruloplasmin and metals in the MPTP model of PD (Fig. 3A). Compared to controls $(n=10)$, MPTP-treated mice $(n=8)$ exhibited elevated iron $(+21 \%$, $P=0.007)$ and copper $(+19 \%, P=0.026)$, accompanying decreased ceruloplasmin levels $(-45 \%$, $P=0.005)$ and activity $(-60 \%, P<0.001)$.

\section{$\underline{\text { Peripherally administered ceruloplasmin enters brain where it confers neuroprotection }}$}

We hypothesized the loss of ceruloplasmin levels after MPTP could contribute to irondependent SN neuronal loss. So we investigated if brain-depletion of ceruloplasmin could be offset by peripheral ceruloplasmin administration and, in turn, rescue the phenotype. $\mathrm{CP}^{-/-}$mice were analyzed for ceruloplasmin entry into perfused brain tissue at intervals over 4 days ( $n=3$ per group) after $5 \mathrm{mg} / \mathrm{kg}$ protein was injected (I.P.). Ceruloplasmin entered the brain with peak 
protein levels occurring after 24 hours (Fig. 3B) demonstrating the communication between brain and peripheral pools of ceruloplasmin. So we investigated whether supplementary ceruloplasmin administration delivered peripherally could rescue the phenotype of a Parkinson model, where ceruloplasmin levels are deficient (Fig. 3A). Control mice $(n=9)$ were compared to two groups of mice administered MPTP. One MPTP-group received ceruloplasmin therapy over 21 days $(n=10)$. The second MPTP-group was administered saline $(n=10)$. Analysis of tissue after the treatment interval revealed that the SN iron elevation in MPTP-mice $(P<0.001)$ was attenuated by peripheral ceruloplasmin treatment $(P=0.01$; Fig. 3C). Neither MPTP nor ceruloplasmin altered liver iron levels (Fig. 3D). Consistent with its effects in preventing nigral iron elevation, ceruloplasmin treatment significantly attenuated both the decrease in striatal dopamine levels $(P=0.005$; Fig. 3E) and the loss of nigral neurons $(P=0.001$; Fig. 3F, G) caused by MPTP.

\section{Discussion}

We provide evidence that the well-documented, moderate decrease of CSF and circulating ceruloplasmin activity in idiopathic $\mathrm{PD}^{13-19}$ may be a reflection of a profound $(\sim 80 \%)$ loss of ceruloplasmin activity in the SN (Fig. 1A,B). Loss of ceruloplasmin activity in SN is important because ceruloplasmin deficiency in $\mathrm{CP}^{-/-}$mice causes $\mathrm{SN}$ neurodegeneration ${ }^{12}$ (Fig. 2D,E) through loss of iron homeostasis (Fig. 2C) and may be the cause of neurodegeneration in aceruloplasminemia $^{11}$. Furthermore, the elevation of nigral iron in the MPTP model of PD could also be contributed by a marked decrease in ceruloplasmin activity (Fig. 3A).

We found that the specific activity, but not protein levels of ceruloplasmin is decreased in PD SN (Fig. 1A,B). Decreased nigral copper (Fig. 1A), which is required for ceruloplasmin enzyme 
activity $^{7}$, is one possible explanation. Alternatively, oxidatively-damaged ceruloplasmin causes copper release and loss of ferroxidase activity in $\mathrm{PD} \mathrm{CSF}^{15}$.

Nigral neurodegeneration in $\mathrm{CP}^{-/-}$mice was attenuated by iron chelation with deferiprone (Fig. 2). Indeed, deferiprone and other iron chelators protect against PD models ${ }^{6,23}$, and deferiprone is currently being tested in a phase II clinical trial for PD (ClinicalTrials.gov identifier: NCT00943748). However, deferiprone and other currently available iron chelators were developed to treat peripheral iron overload, and so off-target iron depletion may be a problem in the treatment of brain disorders. We found that peripheral administration of ceruloplasmin attenuated nigral damage in the MPTP mouse model of PD (Fig. 3E-G), which demonstrated target selectivity by lowering iron in the MPTP-affected SN (Fig. 3C) but not liver (Fig. 3D).

Previous work has shown that ceruloplasmin activity in PD serum is inversely associated with iron deposition in $\mathrm{SN}^{16,19,20}$, and low serum $\mathrm{CP}$ level is a risk factor for $\mathrm{PD}^{17,19,20}$. We found that peripheral ceruloplasmin is in communication with the brain and can cross the blood brain barrier (Fig. 3B). Therefore, the decrease in peripheral ceruloplasmin that occurs in PD may contribute to pathogenesis, or may be a surrogate marker for nigral ceruloplasmin activity (Fig 1A,B). Supplementation of peripheral ceruloplasmin to normal mice was effective in attenuating nigral pathology in the MPTP model (Fig. 3E-G), proving the principle that parenteral ceruloplasmin infusion may be of therapeutic utility in PD. This may warrant investigation in clinical trials.

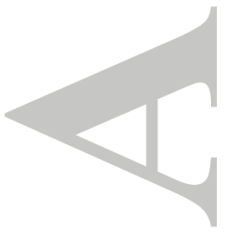




\section{Appendix}

\section{Acknowledgement}

The authors would like to thank the support and advice of Associate Professor Robert Cherny. Supported by funds from the Australian Research Council, the Australian National Health \& Medical Research Council, the CRC for mental health, and Operational Infrastructure Support Victorian State Government. The Victorian Brain Bank Network is supported by The University of Melbourne, The Mental Health Research Institute, The Alfred Hospital and the Victorian Forensic Institute of Medicine, and funded by Neurosciences Australia and the NHMRC.

Competing Interests Drs. Adlard, Finkelstein and Bush are shareholders in and paid scientific consultants for Prana Biotechnology Ltd. Dr. Bush is a shareholder in Eucalyptus Ltd and Cogstate Ltd, and a consultant for Adeona Pharmaceuticals Inc.

\section{References}

1. Oakley AE, Collingwood JF, Dobson J, et al. Individual dopaminergic neurons show raised iron levels in Parkinson disease. Neurology 2007;68:1820-1825.

2. Lei P, Ayton S, Finkelstein DI, et al. Tau deficiency induces parkinsonism with dementia by impairing APP-mediated iron export. Nature medicine 2012;18:291-295.

3. Dexter DT, Carter CJ, Wells FR, et al. Basal lipid peroxidation in substantia nigra is increased in Parkinson's disease. Journal of neurochemistry 1989;52:381-389.

4. Ostrerova-Golts N, Petrucelli L, Hardy J, et al. The A53T alpha-synuclein mutation increases iron-dependent aggregation and toxicity. J Neurosci 2000;20:6048-6054.

5. Castellani RJ, Siedlak SL, Perry G, et al. Sequestration of iron by Lewy bodies in Parkinson's disease. Acta neuropathologica 2000;100:111-114.

6. Molina-Holgado F, Gaeta A, Francis PT, et al. Neuroprotective actions of deferiprone in cultured cortical neurones and SHSY-5Y cells. Journal of neurochemistry 2008;105:2466-2476.

7. Hellman NE, Gitlin JD. Ceruloplasmin metabolism and function. Annual review of nutrition 2002;22:439-458. 
8. Klomp LW, Gitlin JD. Expression of the ceruloplasmin gene in the human retina and brain: implications for a pathogenic model in aceruloplasminemia. Human molecular genetics 1996;5:1989-1996.

9. Duce JA, Tsatsanis A, Cater MA, et al. Iron-export ferroxidase activity of beta-amyloid precursor protein is inhibited by zinc in Alzheimer's disease. Cell 2010;142:857-867.

10. McNeill A, Pandolfo M, Kuhn J, et al. The neurological presentation of ceruloplasmin gene mutations. European neurology 2008;60:200-205.

11. Hochstrasser H, Bauer P, Walter U, et al. Ceruloplasmin gene variations and substantia nigra hyperechogenicity in Parkinson disease. Neurology 2004;63:1912-1917.

12. Patel BN, Dunn RJ, Jeong SY, et al. Ceruloplasmin regulates iron levels in the CNS and prevents free radical injury. J Neurosci 2002;22:6578-6586.

13. Boll MC, Alcaraz-Zubeldia M, Montes S, et al. Free copper, ferroxidase and SOD1 activities, lipid peroxidation and $\mathrm{NO}(\mathrm{x})$ content in the CSF. A different marker profile in four neurodegenerative diseases. Neurochemical research 2008;33:1717-1723.

14. Boll MC, Sotelo J, Otero E, et al. Reduced ferroxidase activity in the cerebrospinal fluid from patients with Parkinson's disease. Neuroscience letters 1999;265:155-158.

15. Olivieri S, Conti A, Iannaccone S, et al. Ceruloplasmin oxidation, a feature of Parkinson's disease CSF, inhibits ferroxidase activity and promotes cellular iron retention. J Neurosci 2011;31:18568-18577.

16. Martinez-Hernandez R, Montes S, Higuera-Calleja J, et al. Plasma ceruloplasmin ferroxidase activity correlates with the nigral sonographic area in Parkinson's disease patients: a pilot study. Neurochemical research 2011;36:2111-2115.

17. Bharucha KJ, Friedman JK, Vincent AS, et al. Lower serum ceruloplasmin levels correlate with younger age of onset in Parkinson's disease. Journal of neurology 2008;255:19571962.

18. Torsdottir G, Kristinsson J, Sveinbjornsdottir S, et al. Copper, ceruloplasmin, superoxide dismutase and iron parameters in Parkinson's disease. Pharmacology \& toxicology 1999;85:239243.

19. Jin L, Wang J, Zhao L, et al. Decreased serum ceruloplasmin levels characteristically aggravate nigral iron deposition in Parkinson's disease. Brain : a journal of neurology 2011;134:50-58.

20. Jin L, Wang J, Jin H, et al. Nigral iron deposition occurs across motor phenotypes of Parkinson's disease. European journal of neurology : the official journal of the European Federation of Neurological Societies 2012. 
21. Torsdottir G, Sveinbjornsdottir S, Kristinsson J, et al. Ceruloplasmin and superoxide dismutase (SOD1) in Parkinson's disease: a follow-up study. Journal of the neurological sciences 2006;241:53-58.

22. Rozas G, Lopez-Martin E, Guerra MJ, et al. The overall rod performance test in the MPTP-treated-mouse model of Parkinsonism. Journal of neuroscience methods 1998;83:165175.

23. Kaur D, Yantiri F, Rajagopalan S, et al. Genetic or pharmacological iron chelation prevents MPTP-induced neurotoxicity in vivo: a novel therapy for Parkinson's disease. Neuron 2003;37:899-909.
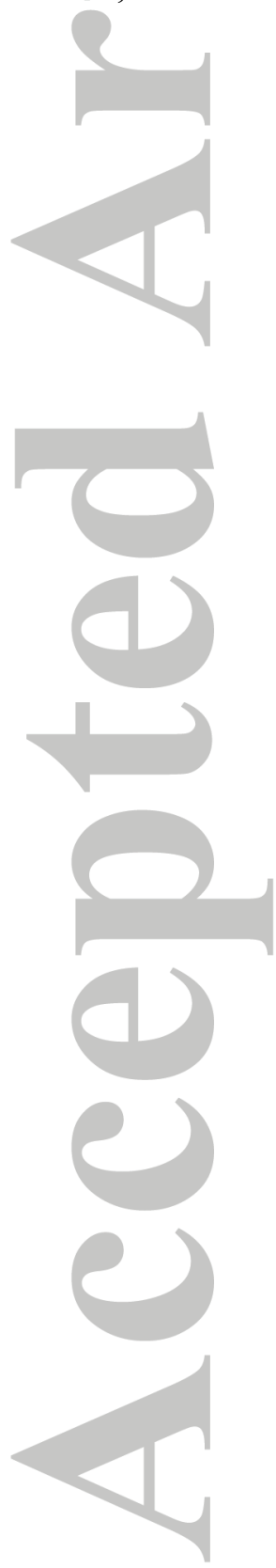


\section{Figure Legends}

Figure 1. Reduced ceruloplasmin (CP) specific activity in PD SN. (A) Iron, copper, ceruloplasmin (CP) levels and CP ferroxidase activity in SN tissue from clinically- and pathologically-characterized PD cases $(n=10)$ compared with $\mathrm{SN}$ from age-matched control cases $(\mathrm{n}=10)$. In PD SN, iron was increased $(P=0.021)$, copper decreased $(P=0.003)$, CP levels unchanged and CP activity (Azide inhibited fraction of transferrin assay) was decreased $(P<0.001)$. (B) CP-specific ferroxidase activity (transferrin assay) was additionally determined by CP immunoprecipitated of selected control $(n=5)$ and PD $(n=7)$ cases. Ferroxidase signal of sham immunopreicpiated samples (designated with minus sign) were subtracted by the ferroxidase signal of CP immunodepleted samples (designated with plus sign). The CP-specific ferroxidase activity was decreased in PD samples compared to controls $(P=0.03)$. (C) Frontal cortices from the same cases were analyzed, but metal levels, CP levels and activity, were unaltered. Representative western blots are shown. Data are means \pm SEM. $* P<0.05, * * P<0.01$, $* * * P<0.001$, two-tailed $t$-tests.

Figure 2. Iron chelation rescues Parkinsonism in $\mathbf{C P}^{-/-}$mice. 6-month old WT mice $(n=10)$ were compared with 6-month old $\mathrm{CP}^{-/-}$mice $(\mathrm{n}=8)$, and $\mathrm{CP}^{-/-}$mice administered deferiprone in their drinking water for 3 months $(n=9)$. (A) Mice were tested for motor ability with the rotarod (Two-way $A N O V A ; P=0.045)$. Simple main effects: $\mathrm{CP}^{-/-}$mice exhibited significantly reduced latency to fall $(P=0.043)$, which was rescued by treatment with deferiprone $(P=0.02)$. (B) Performance of mice on the accelerating rotarod was studied by Kaplan-Meier survival analysis. Mantel-cox pairwise comparisons demonstrated that non-treated $\mathrm{CP}^{-/-}$mice had reduced speed survival $(P=0.025)$, which was restored by deferiprone $(P=0.05)$. (C) The right $\mathrm{SN}$ was assayed 
for iron (Two-way $A N O V A, P=0.01$ ). Simple main effects: iron in $\mathrm{CP}^{-/-}$nigra is elevated $(P=0.008)$, but normalized by deferiprone treatment $(P=0.007)$. (D) $\mathrm{SN}$ neuron number was assayed by unbiased stereology (Two-way $A N O V A, P<0.001$ ). Simple main effects: non-treated $\mathrm{CP}^{-/-}$mice had fewer SN neurons compared to WT $(P<0.001)$, which was partially rescued by deferiprone $(P=0.002)$. (E) Representative sections displaying loss and rescue of SN neurons (Nissl stain). Scale bar $250 \mu \mathrm{m}$. Data are means \pm SEM. ${ }^{*} P<0.05,{ }^{*} P<0.01, * * * P<0.001$.

Figure 3. Peripheral administration of ceruloplasmin (CP) enters the brain where it confers neuroprotection against MPTP. (A) The SN was microdissected from MPTP-treated (n=8) and compared with non-treated controls $(n=10)$. Metal levels were measured, as well as CP levels and activity and compared by $t$-tests. MPTP caused elevated iron $(P=0.007)$ and copper $(P=0.026)$ following MPTP, while CP levels $(P=0.005)$ and activity $(P<0.001)$ decreased. (B) $5 \mathrm{mg} / \mathrm{kg} \mathrm{CP}$ was administered I.P. to $\mathrm{CP}^{-/-}$mice. Mice were euthanized at time points indicated and their brains were analyzed for CP protein by western blot. (C) Two groups of C57/B16 mice were administered MPTP, one group was then treated further with supplementary CP $(5 \mathrm{mg} / \mathrm{kg}$, I.P.; $n=10)$ while the other was administered saline $(n=10)$. These were compared against nonlesioned controls $(\mathrm{n}=9)$. The right $\mathrm{SN}$ was dissected 21 days after lesion. SN iron differed between groups (two-way $A N O V A, P<0.001)$. Simple main effects: $\mathrm{SN}$ iron was elevated by MPTP (relative to control, $P<0.001)$, but prevented by co-administration of $\mathrm{CP}(P=0.01)$. (D) Iron was not altered in liver across conditions. (E) CP treatment rescued the decrease in striatal dopamine caused by MPTP ( $t$-test, $P=0.005)$. (F) The left SN was sectioned for unbiased stereological estimation of SN neurons (two-way $A N O V A, P<0.001$ ). Simple main effects:

MPTP caused a reduction in SN neurons $(P<0.001)$ that was partially rescued by treatment with 
$\mathrm{CP}(P=0.001)$. (G) Representative sections displaying loss and rescue of SN neurons (Nissl stain). Scale bar $250 \mu \mathrm{m}$. Data are means \pm SEM. ${ }^{*} P<0.05,{ }^{* *} P<0.01, * * * P<0.001$.

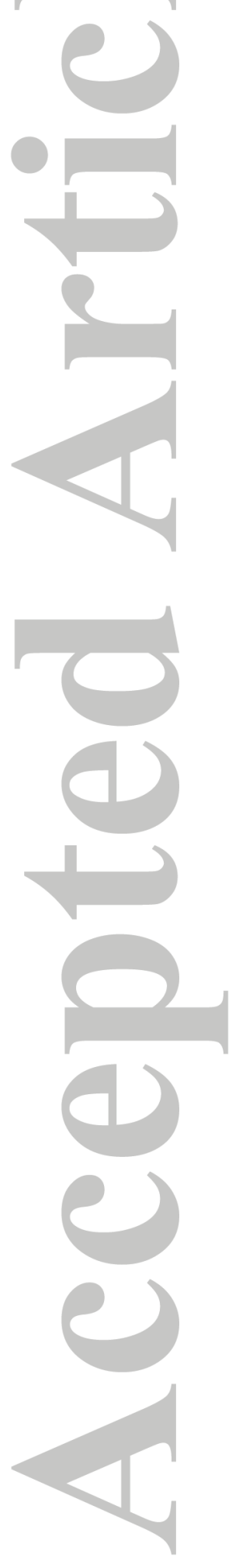




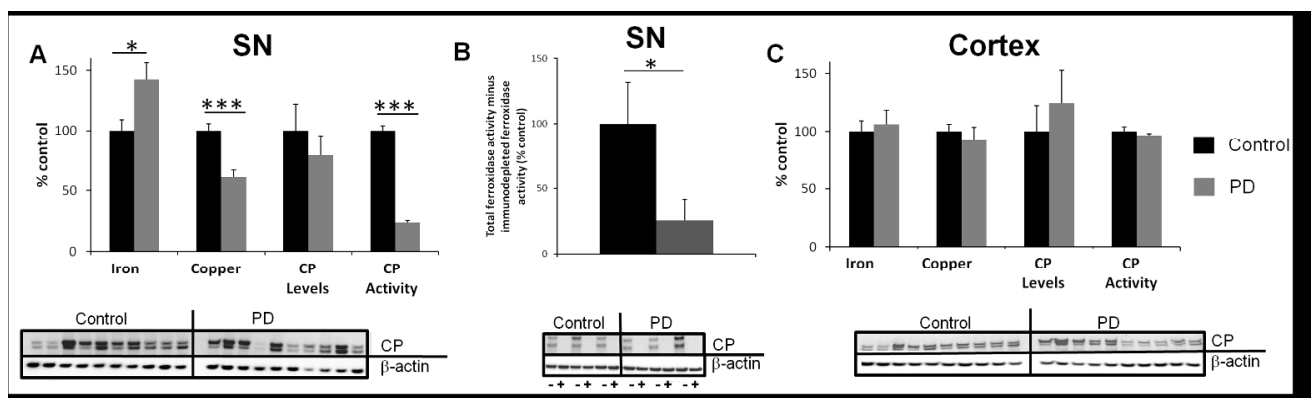

Figure 1. Reduced ceruloplasmin (CP) specific activity in PD SN. (A) Iron, copper, ceruloplasmin (CP) levels and CP ferroxidase activity in SN tissue from clinically- and pathologically-characterisedcharacterized PD cases $(n=10)$ compared with SN from age-matched control cases $(n=10)$. In PD SN, iron was increased $(P=0.021)$, copper decreased $(P=0.003), C P$ levels unchanged and $C P$ activity was decreased $(P<0.001)$. ( $B$ (Azide inhibited fraction of transferrin assay) was decreased $(P<0.001)$. (B) CP-specific ferroxidase activity (transferrin assay) was additionally determined by CP immunoprecipitated of selected control ( $n=5$ ) and PD $(n=7)$ cases. Ferroxidase signal of sham immunopreicpiated samples (designated with minus sign) were subtracted by the ferroxidase signal of CP immunodepleted samples (designated with plus sign). The $C P$-specific ferroxidase activity was decreased in PD samples compared to controls $(P=0.03)$. (C) Frontal cortices from the same cases were analyzed, but metal levels, CP levels and activity, were unaltered. Representative western blots are shown. Data are means $\pm \mathrm{SEM} . * \mathrm{P}<0.05, * * \mathrm{P}<0.01, * * * \mathrm{P}<0.001$, twotailed t-tests.

\section{$436 \times 129 \mathrm{~mm}$ (150 x 150 DPI)}




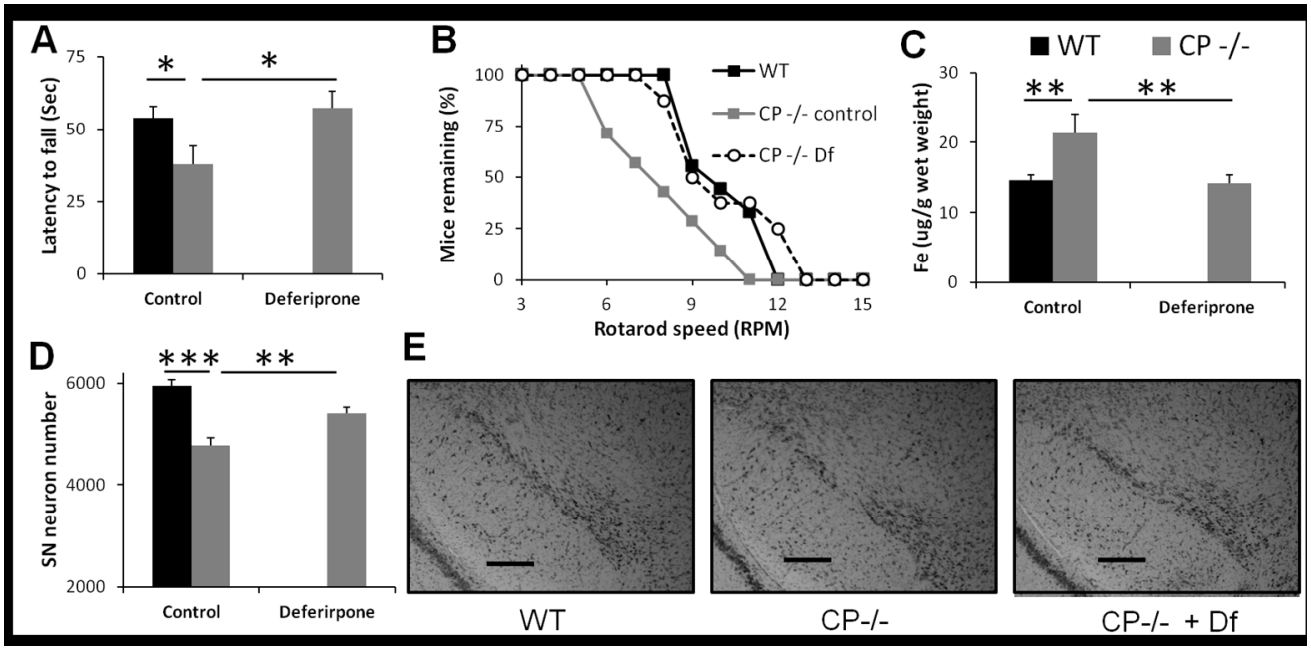

Figure 2. Iron chelation rescues Parkinsonism in CP-/- mice. 6-month old WT mice $(n=10)$ were compared with 6-month old $\mathrm{CP}$-/- mice $(\mathrm{n}=8)$, and $\mathrm{CP}$-/- mice administered deferiprone in their drinking water for 3 months $(n=9)$. (A) Mice were tested for motor ability with the rotarod (Two-way ANOVA; $P=0.045$ ). Simple main effects: $C P-/-$ mice exhibited significantly reduced latency to fall $(P=0.043)$, which was rescued by treatment with deferiprone $(P=0.02)$. (B) Performance of mice on the accelerating rotarod was studied by Kaplan-Meier survival analysis. Mantel-cox pairwise comparisons demonstrated that non-treated CP-/- mice had reduced speed survival $(P=0.025)$, which was restored by deferiprone $(P=0.05)$. (C) The right $S N$ was assayed for iron (Two-way ANOVA, $P=0.01$ ). Simple main effects: iron in $C P-/-$ nigra is elevated $(P=0.008)$, but normalized by deferiprone treatment $(P=0.007)$. (D) $S N$ neuron number was assayed by unbiased stereology (Two-way ANOVA, P<0.001). Simple main effects: non-treated $\mathrm{CP}-/-$ mice had fewer SN neurons compared to WT $(P<0.001)$, which was partially rescued by deferiprone $(P=0.002)$. (E) Representative sections displaying loss and rescue of SN neurons (Nissl stain). Scale bar $250 \mathrm{~mm}$. Data are means \pm SEM. $* \mathrm{P}<0.05, * * \mathrm{P}<0.01, * * * \mathrm{P}<0.001$. $287 \times 142 \mathrm{~mm}(150 \times 150 \mathrm{DPI})$

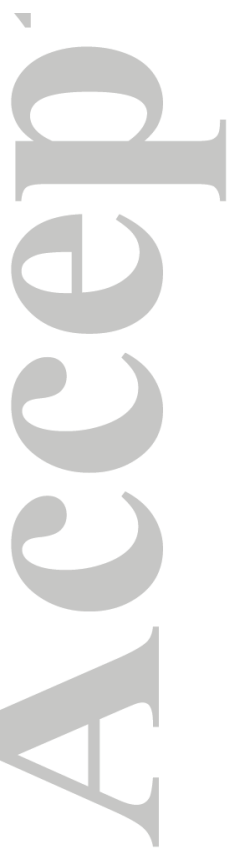




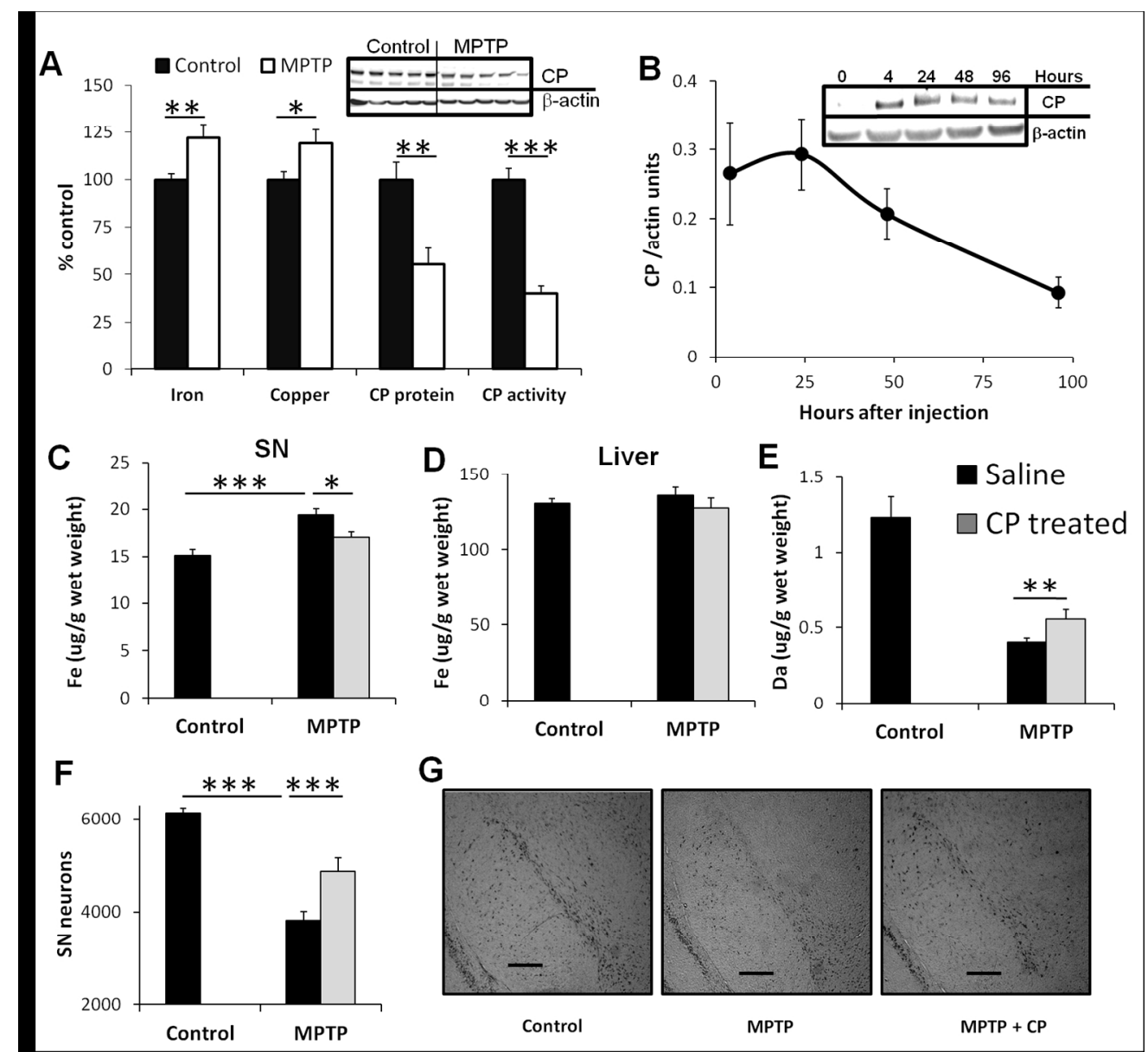

Figure 3. Peripheral administration of ceruloplasmin (CP) enters the brain where it confers neuroprotection against MPTP. (A) The SN was microdissected from MPTP-treated $(n=8)$ and compared with non-treated controls $(n=10)$. Metal levels were measured, as well as CP levels and activity and compared by $t$-tests. MPTP caused elevated iron $(P=0.007)$ and copper $(P=0.026)$ following MPTP, while CP levels $(P=0.005)$ and activity $(P<0.001)$ decreased. (B) $5 \mathrm{mg} / \mathrm{kg}$ CP was administered I.P. to CP-/- mice. Mice were euthanized at time points indicated and their brains were analyzed for CP protein by western blot. (C) Two groups of C57/BI6 mice were administered MPTP, one group was then treated further with supplementary CP (5mg/kg, I.P.; $n=10)$ while the other was administered saline $(n=10)$. These were compared against non-lesioned controls $(n=9)$. The right SN was dissected 21 days after lesion. SN iron differed between groups (two-way ANOVA, $\mathrm{P}<0.001$ ). Simple main effects: SN iron was elevated by MPTP (relative to control, $\mathrm{P}<0.001$ ), but prevented by co-administration of $C P(P=0.01)$. (D) Iron was not altered in liver across conditions. $(E) C P$ treatment rescued the decrease in striatal dopamine caused by MPTP ( $t$-test, $P=0.005)$. ( $F$ ) The left $S N$ was sectioned for unbiased stereological estimation of SN neurons (two-way ANOVA, $P<0.001$ ). Simple main effects: MPTP caused a reduction in $S N$ neurons $(P<0.001)$ that was partially rescued by treatment with $C P$ $(P=0.001)$. ( $G)$ Representative sections displaying loss and rescue of SN neurons (Nissl stain). Scale bar 250 $\mathrm{mm}$. Data are means \pm SEM. $* \mathrm{p}<0.05, * * \mathrm{P}<0.01, * * * \mathrm{p}<0.001$.

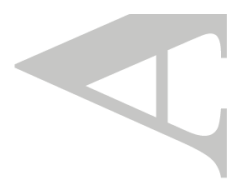

$282 \times 260 \mathrm{~mm}(150 \times 150 \mathrm{DPI})$ 


\section{Supplementary material}

\section{Ceruloplasmin dysfunction and therapeutic potential for Parkinson's}

\section{disease}

Scott Ayton $\mathrm{PhD}^{1}$, Peng Lei $\mathrm{PhD}^{1}$, James A. Duce, $\mathrm{PhD}^{1}$, Bruce X. W. Wong, $\mathrm{PhD}^{1}$, Amelia Sedjahtera BBMS(Hons) ${ }^{1}$, Paul A. Adlard $\mathrm{PhD}^{2,3}$, Ashley I. Bush MBBS $\mathrm{PhD}^{1,3,{ }^{*}}$ and David I. Finkelstein, $\mathrm{PhD}^{1,2}$

${ }^{1}$ Oxidation Biology Laboratory and ${ }^{2}$ Synaptic Neuropathology, Florey Institute for Neuroscience and Mental Health, and ${ }^{3}$ Department of Pathology, The University of Melbourne, Victoria, Australia.

*Correspondence to: ashleyib@unimelb.edu.au

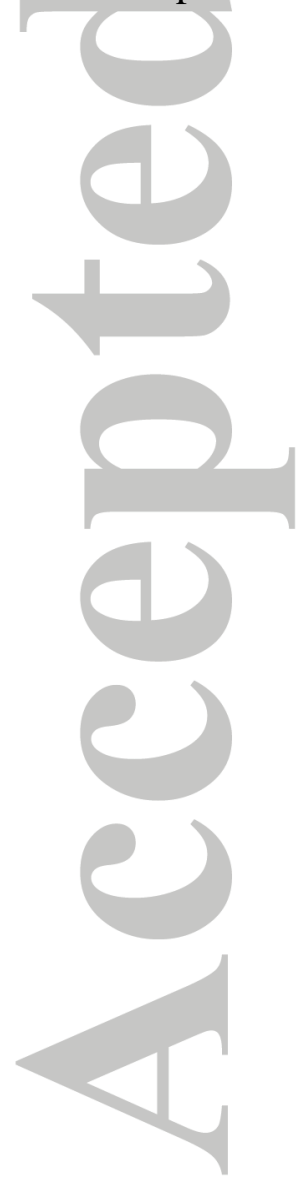


Supplementary Table 1: Clinicopathological information for human cases.

\begin{tabular}{|l|l|l|l|l|}
\hline Diagnosis & Age & Sex & Post mortem interval (h) & Cause of Death \\
\hline Control & 72 & Male & 42.5 & Ischemic heart disease \\
\hline Control & 75 & Male & 46 & Ruptured abdominal aortic aneurysm \\
\hline Control & 75 & Female & 22.5 & Lobar pneumonia \\
\hline Control & 85 & Female & 71 & Multi-organ failure \\
\hline Control & 63 & Female & 30 & Pulmonary embolism \\
Control & 82 & Female & 57.5 & $\begin{array}{l}\text { Cardiac tapenade, ruptured aortic arch } \\
\text { aneurysm }\end{array}$ \\
\hline Control & 73 & Male & 49 & Ischemic heart disease \\
\hline Control & 84 & Male & 55 & Ischemic heart disease \\
\hline Control & 77 & Male & 53.6 & Acute myocardial infarction \\
\hline Control & 80 & Female & 59 & Cardiac failure \\
\hline PD & 71 & Male & 56 & Cardiac arrest \\
\hline PD & 74 & Male & 24.5 & Stroke \\
\hline PD & 76 & Female & 12 & Cardiac arrest \\
\hline PD & 84 & Female & 34 & Stroke \\
\hline PD & 84 & Male & 50.5 & Pneumonia \\
\hline PD & 74 & Male & 23 & Ischemic heart disease \\
\hline PD & 63 & Female & 56 & Ischemic heart disease \\
\hline PD & 78 & Female & 20.5 & Ischemic Heart Disease \\
\hline PD & 82 & Female & 26 & Cardiac arrest \\
\hline PD & 72 & Male & 45 & Pulmonary thromboembolism \\
\hline
\end{tabular}




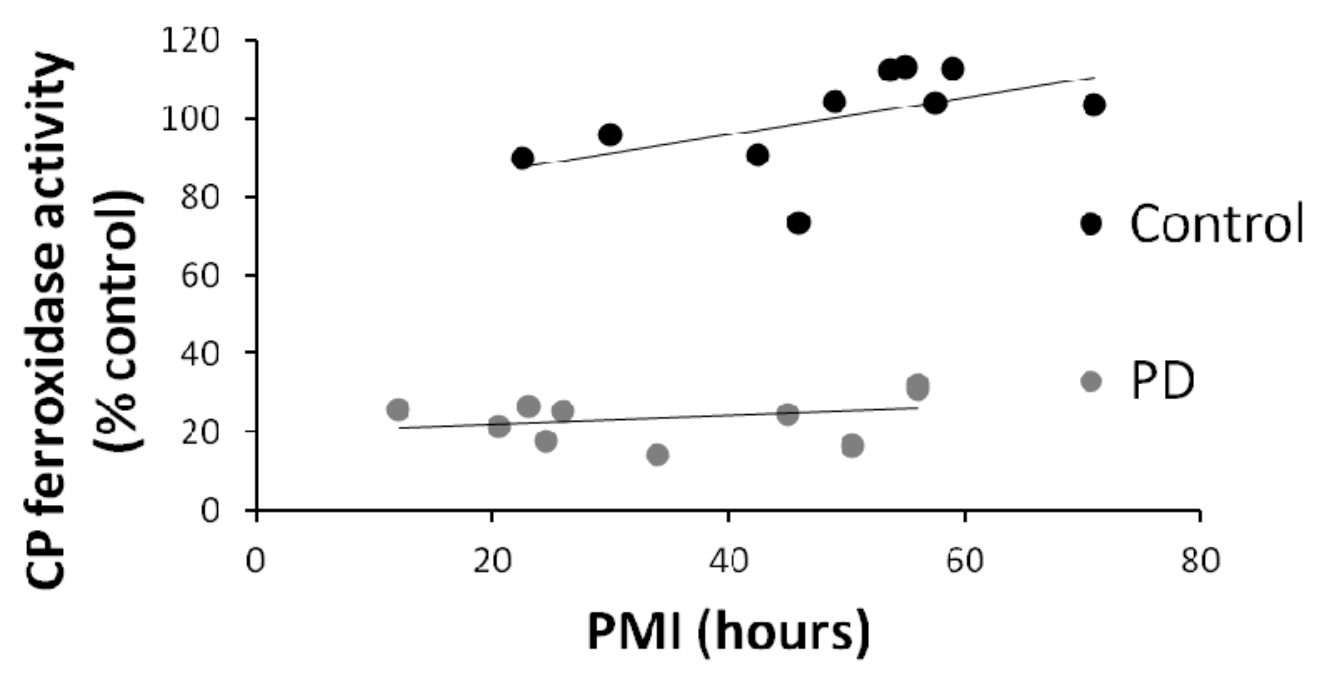

B

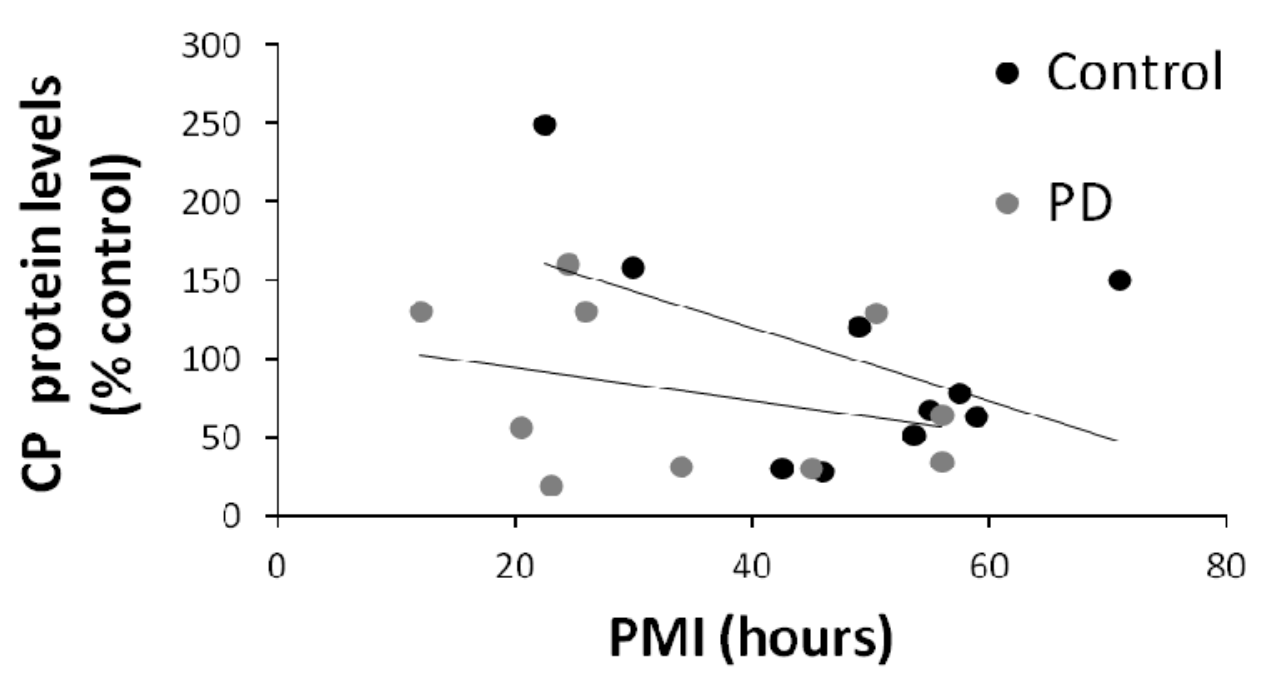

Supplementary Figure 1. No association between post mortem interval (PMI) and either ceruloplasmin (CP) level or activity. (A) CP ferroxidase activity was measured as per Fig 1. Pearson's regression between CP ferroxidase activity and PMI showed no significant association in controls $\left(r^{2}=0.27, \mathrm{p}=0.116\right)$ and $\mathrm{PD}\left(r^{2}=0.07, P=0.43\right)$ post mortem SN. (B) CP protein level was measured as per Fig 1. Pearson's regression between CP protein level and PMI showed no significant association in controls $\left(r^{2}=0.22, P=0.164\right)$ and $\operatorname{PD}\left(r^{2}=0.09\right.$, $\mathrm{p}=0.38$ ) post mortem $\mathrm{SN}$. 


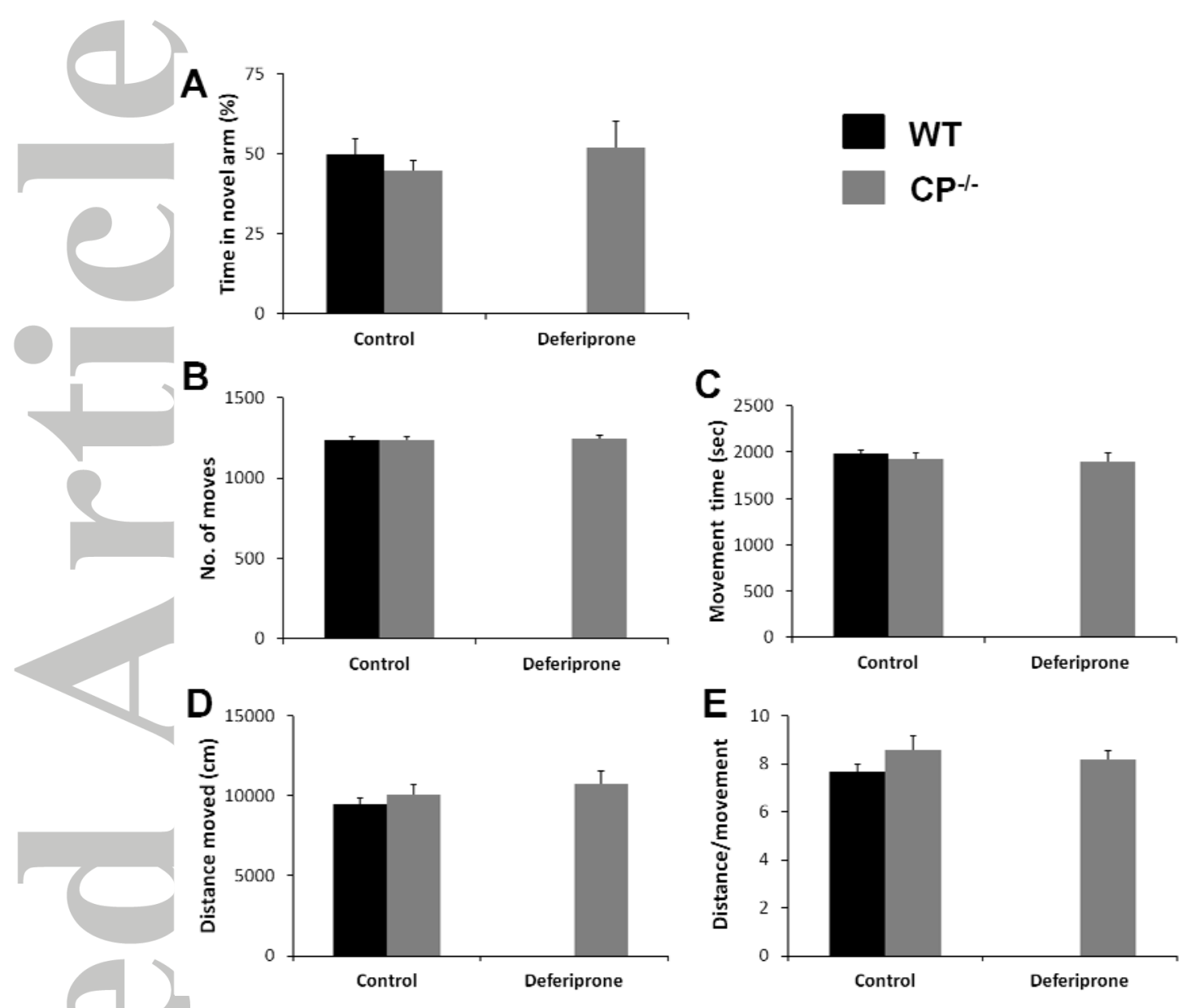

Supplementary Figure 2. No change in Y-maze or open field behaviour in WT and $\mathrm{CP}^{-/-}$ mice at 6 months of age. 6 -month old $\mathrm{WT}$ and $\mathrm{CP}^{-/-}$mice treated \pm deferiprone for 3 months (as per Fig 2). (A) For Y-maze, there was no difference in time spent in novel arm indicating that spatial memory was not affected in any condition. (B-E) Open Field analysis was performed on all mice. No Change was observed in any condition for total number of moves (B), total time in movement (C), total distance moved (D) and the distance moved per movement (E). Data are means \pm SEM. 

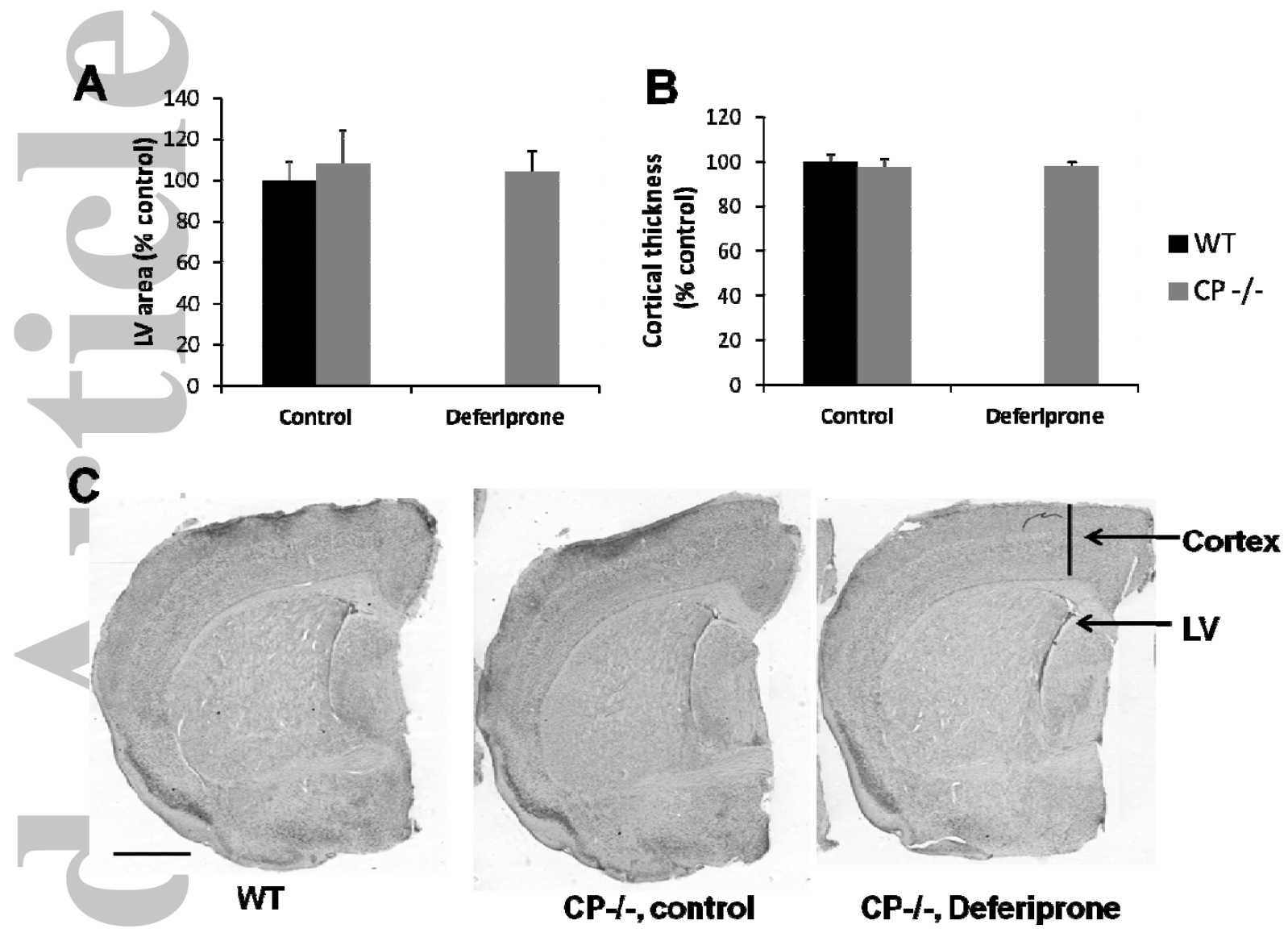

Supplementary Figure 3. No Change in Lateral ventricular area or cortical thickness in WT and $\mathrm{CP}^{-/-}$mice at 6 months of age. 6-month old WT and $\mathrm{CP}^{-/-}$mice treated \pm deferiprone for 3 months (as per Fig. 2). Nissl-stained coronal sections through the anterior commissure were used to measure (A) lateral ventricular (LV) area and (B) cortical thickness. (C) Representative sections are show. Scale bar equals $1 \mathrm{~mm}$. Data are means $\pm \mathrm{SEM}$. 


\section{Supplementary methods}

\section{$\underline{\text { Rotarod }}$}

Mice were placed on a rotating rod (Panlab, Barcelona, Spain), spinning at 4 RPM (Lane width, 50mm, rod diameter $30 \mathrm{~mm}$ ). Once stabilised, mice were subjected to an incrementally increasing speed of 1 RPM per 8 seconds. Each animal underwent 3 trials. The length of time that the mice managed to remain on the rod, and the speed at which they fell off the apparatus, were recorded. The average of the three trials was used for further analysis.

\section{$\underline{\text { Y-maze }}$}

The Y maze test utilizes a Y-shaped grey-painted timber with arms $29.5 \mathrm{~cm}$ long $\times 7.5 \mathrm{~cm}$ wide $\times 15.5 \mathrm{~cm}$. All mice were subjected to a 2-trial Y-maze test separated by a 1-h inter-trial interval to assess spatial memory, with all testing performed during the light phase of the circadian cycle. The 3 identical arms were randomly designated start arm, novel arm, and other arm. Visual cues were placed on the walls of the maze. The first trial (training) was for 10 min, and the mice were allowed to explore only 2 arms (starting arm and other arm). For the second trial (retention) mice were placed back in the maze in the same starting arm, and allowed to explore for 5 min with free access to all 3 arms. Behaviours were recorded on video during a 1 min trial and Ethovision video-tracking system (Noldus, Netherlands) was used for analysis. The amount of time spent in the novel arm is indicative of spatial memory. Where mice exhibit spatial memory impairment, the amount of time spent in the novel arm should approximate the amount of time spent in each of the other two arms (therefore, $33 \%$ in each arm). Data are expressed as the percentage of duration mice spent in the novel during the 1-min second trial.

\section{Open Field}


For the Open Field Test, spontaneous motor activity of mice was measured using a photobeam activity system (Truscan 2.0, Coulbourn Instruments). The test area was $25.4 \mathrm{~cm}$ wide by $25.4 \mathrm{~cm}$ deep by $40.64 \mathrm{~cm}$ high. The mouse was placed in the chamber for one hour to allow acclimatization to its surroundings. Parameters of movements were calculated from the interception of beams to provide an XY coordinate.

\section{$\underline{\text { Stereological estimation of nigral neurons }}$}

Nissl-stained substantia nigra pars compactor (SNpc) neurons were counted by blinded and unbiased stereology. The left SN was removed for estimation of nigral neuron number using by blinded and unbiased stereology using our previously described methods ${ }^{1-6}$. Briefly, the midbrain was micro-dissected and then cut $(30 \mu \mathrm{m})$ in 1:3 series with a cryostat, then stained with neutral red (Grale Scientific, Victoria, Australia). The neurons in the SNpc were counted according to the optical fractionator rules, and the neurons in the SNpc were distinguished by the anatomical location, orientation, presence of nucleolus and cell density. Neuron numbers were estimated using an unbiased counting frame of $x=35 \mu \mathrm{m}, \mathrm{y}=45 \mu \mathrm{m}$ $\left(1575 \mu \mathrm{m}^{2}\right)$ at regular intervals on a sampling grid of $140 \mathrm{x} 140(\mathrm{x}=140 \mu \mathrm{m}, \mathrm{y}=140 \mu \mathrm{m})$, and a stereological program (Stereoinvestigator, Microbrightfield, USA) viewed with a $60 \times 1.3$ N.A. oil objective (DMLB Leica Microscope).

\section{$\underline{\text { Lateral ventricular area and cortical thickness }}$}

The left hemisphere was sectioned through the forebrain $(30 \mathrm{~mm})$ and sections were stained with Nissel. Using the anterior commissure as a landmark, the cortical thickness and lateral ventricular area were measured using ImageJ software, as per our previous study ${ }^{7}$.

\section{$\underline{\text { Western blot }}$}

Sample homogenate was separated by $4-12 \%$ bis-tris gels (Invitrogen) and electroblotted on a nitrocellulose membrane (iBlot; Invitrogen). Ceruloplasmin antibody (Vital diagnostics) 
was imaged by enhanced chemiluminescence (GE Healthcare) and scanned with Fujifilm LAS-3000. Membranes were re-probed with $\beta$-actin antibody (Sigma).

\section{$\underline{\text { Dopamine measurement }}$}

The right striatum of mice was microdissected for dopamine analysis. This tissue homogenised by pulse sonication in $300 \mu \mathrm{l}$ of $0.4 \mathrm{M}$ perchloric acid (Sigma Aldrich), $0.15 \%$ sodium metabisulphite (Sigma Aldrich), and 0.05\% Ethylenediaminetetra acetic acid disodium salt (EDTA) (Sigma Aldrich). The homogenates were centrifuged and the supernatant collected for dopamine measurement with HPLC coupled to an electrochemical detection system (ESA Coulochem III). Briefly, 20 $\mu$ l supernatant samples were injected onto a MD-150 reverse phase C18 column. The system was equilibrated at $0.6 \mathrm{ml} / \mathrm{min}$ with the mobile phase consisting of 75mM sodium dihydrogen phosphate, monohydrate (Sigma), 1.7mM 1-Octanesulfonic Acid sodium salt (Sigma), 100 $\mu \mathrm{l} / \mathrm{l}$ Triethylamine, $25 \mu \mathrm{M}$ EDTA (Sigma) in 10\% acetonitrile, pH 3. Compounds were detected and quantified with an electrochemical detector with a conditioning cell (E1:-150mV, E2:+220mV, and guard cell: $+250 \mathrm{mV}$ ). Peaks were identified by the retention time of a dopamine standard (Aigma). Data were normalised to total wet weight of sample.

\section{Atomic absorption spectroscopy (AAS)}

Homogenized tissue was lyophilised, digested (69\% nitric acid, 30\% hydrogen peroxide), then metal assayed by a Varian AA240 AAS. Triplicate measurements were standardized to wet weight.

\section{$\underline{\text { Statistics }}$}

Statistical analysis was performed with SPSS software. Details can be found throughout the text. 


\section{References}

1. Parish CL, Finkelstein DI, Drago J, et al. The role of dopamine receptors in regulating the size of axonal arbors. J Neurosci 2001;21:5147-5157.

2. Finkelstein DI, Stanic D, Parish CL, et al. Axonal sprouting following lesions of the rat substantia nigra. Neuroscience 2000;97:99-112.

3. Hardman CD, Henderson JM, Finkelstein DI, et al. Comparison of the basal ganglia in rats, marmosets, macaques, baboons, and humans: volume and neuronal number for the output, internal relay, and striatal modulating nuclei. The Journal of comparative neurology 2002;445:238-255.

4. Lee J, Zhu WM, Stanic D, et al. Sprouting of dopamine terminals and altered dopamine release and uptake in Parkinsonian dyskinaesia. Brain 2008;131:1574-1587.

5. Parish CL, Drago J, Stanic D, et al. Haloperidol treatment reverses behavioural and anatomical changes in cocaine-dependent mice. Neurobiology of disease 2005;19:301-311. 6. Stanic D, Finkelstein DI, Bourke DW, et al. Timecourse of striatal re-innervation following lesions of dopaminergic SNpc neurons of the rat. The European journal of neuroscience 2003;18:1175-1188.

7. Lei P, Ayton S, Finkelstein DI, et al. Tau deficiency induces parkinsonism with dementia by impairing APP-mediated iron export. Nature medicine 2012;18:291-295. 